\title{
ORIGINAL
}

\section{EVOLUCIÓN DE LA PREVALENCIA DE INFECCIÓN POR VIRUS DE LA INMUNODEFICIFNCIA HUMANA EN POBLACIÓN RECLUSA AL INGRESO EN PRISIÓN DURANTE EL PERÍODO 1991-1995*}

Vicente Martín (1), Joan A. Caylà (2), María L. Morís (3), Luis E. Alonso (1) y Rafael Pérez (3).

(1) Servicio Médico. Centro Penitenciario. León.

(2) Servicio de Epidemiología. Instituto Municipal de la Salud. Barcelona.

(3) Servicio de Análisis Clínicos. Hospital de León.

* Este trabajo ha sido parcialmente financiado por una ayuda de investigación del Fondo de Investigación Sanitaria (N. ${ }^{\circ}$ de expediente $\left.96 / 1524\right)$.

\section{RESUMEN}

Fundamento: Las prisiones españolas albergan un elevado número de usuarios de drogas por vía parenteral (UDVP), y otras personas con prácticas de riesgo para la infección por virus de la inmunodeficiencia humana (VIH). El objetivo de este trabajo es conocer la evolución de la prevalencia de infección por VIH en el momento del ingreso en prisión y los factores asociados a la misma en este colectivo, lo que puede permitir una aproximación a la efectividad de las estrategias de reducción de riesgos y contribuir a mejorarlas.

Métodos: Todas aquellas personas que ingresaron en un centro penitenciario provincial del noroeste español entre los años 1991 y 1995. Se recogieron variables socio-demugráficas, penitenciarias y factores de riesgo para VIH. Previo consentimiento. se practicó prueba de infección por VIH (ELISA y Western-blot).

Resultados: El 19,4\% de los 1663 individuos estudiados estaban infeclados por VIH. Destacaban, con significación estadística. las prevalencias en: mujeres $(26.0 \%)$, el grupo de edad desde 25 a 34 anos $(29.1 \%)$. blancos $(20,9 \%)$, solteros $(22,8 \%)$. tatuados $(29.9 \%)$. los que presentaban antecedentes de autolesiones $(42.4 \%)$, los UDVP $(46,3 \%)$. los que reconocieron compartir jeringuillas $(61,5 \%)$ y aquellos con antecedentes de haber permanecido en prisión un año o más $(37,3 \%)$. El análisis de regresión logística mostró como predictores de infección VIH: los UDVP. los que ingresaron en 1992, las mujeres, los grupos de edad de 25-34 y de 35-44 años. los tatuados, los que presintaban antecedentes de autolesiones y aquéllos con antecedentes de estancia en prisión superior a un año. La etnia gitana presento menor probabilidad de infección por VIH.

La tendencia temporal de la infección VIH. estratificada según el antecedente de estancia previa en prisión, mostró un descenso casi significativo $(P=0.064)$. La tendencia de la infección según UDVP no mostró modificación $(\mathrm{P}=0,16)$.

Conclusiones: Se ha detectado una elevada prevalencia de infección por VIH en una prisión de una región poco afectada por SIDA. EI UDVP y algunas características relacionables con este colectivo influyen enormemente en este fenómeno. La tendencia temporal de esta infección en el colectivo ha podido disminuir por la menor proporción de UDVP que ingresan en prision. si bien en este grupo se han mantenido prevalencias muy elevadas de infección a lo largo de los cinco años de estudio. Se recomienda impulsar en prisiones programas de reducción de riesgos (programas de mantenimiento con metadona. programas piloto de intercambio de jeringuillas).

Palabras clave: VIH. Prisión. Usuarios drogas vía parenteral. Epidemiología. Salud pública.

Correspondencia:

Vicente Martín Sánchez.

Juan de Malinas, 2. 4. ${ }^{\circ} \mathrm{B}$.

24005 LEÓN.

\section{ABSTRACT \\ Evolution of the Prevalence of the Human Immunodeficiency Virus among Inmates on their Admission to Prison during the Period 1991-1995}

Background: Inmates of Spanish prisons include a high number of intravenous drug users (IVDUs) and other people whose practices entail the risk of infection with the human immunodeficiency virus (HIV). The aim of this work is to find out the evolution of the prevalence of HIV infection at the time of admission to prison and the factors associated with it in this population group. This may enable us to form an idea of the effectiveness of risk reduction strategies and help to improve them.

Methods: All those people who were placed in a provincial penal institution in the northwest of Spain between 1991 and 1995. Socio-demographic, penal and HIV risk factor variables were gathered. The HIV infection test (ELISA and Westernblot) was carried out with the consent of the subjects.

Results: Of the 1.663 people studied, $19.4 \%$ were HIV-positive. The prevalence HIV infection was particularly marked statistically in: women $(26.0 \%)$, the 25-34 age group (29.1\%). whites $(20.9 \%)$, single people $(22.8 \%)$, those people with a tattoo $(29.9 \%)$, those people with a background in self-inflicted injuries $(42.2 \%)$, IVDUs $(46.3 \%)$, those who admitted sharing syringes $(61.5 \%)$ and those with a prison record of one or more years $(37.3 \%)$. Logistical regression analysis showed the following as prediclors of HIV infection: IVDUs, those who went to prison in 1992, women, the 25-34 and 35-44 age group, tattooed men, those with a background in self-inflicted injuries and those with a prison record, of more than one year. The gypsy ethnic group revealed a lower probability of HIV infection.

The HIV infection time trend, stratified according to the prison record showed an almost significant drop $(\mathrm{P}=0.064)$. The infection trend per IVDU did not show any modification $(\mathrm{P}=0.16)$.

Conclusions: A high prevalence of HIV infection was detected in a prison located in a region which has not been particularly affected by AIDS. IVDUs and some characteristics that may be related to this population group have an enormous influence on this phenomenon. The time trend for this infection in this population group has decreased through the lower number of IVDUs that are admitted to prison although very high levels of prevalence of the infection were maintained in this group over the five years of the study. It is recommended that risk-reduction programmes in prisons be fostcred (methadone maintenance programmes, syringe exchange pilot programmes).

Key words: HIV. Prison. Intravenous drug users. Epidemiology. Public health. 


\section{INTRODUCCIÓN}

La infección por virus de la inmunodeficiencia humana (VIH) es probablemente el mayor problema de salud pública en los sistemas penitenciarios europeos y de los Estados Unidos de América ${ }^{1,2}$. En la población reclusa española han sido descritas prevalencias de infección por VIH muy elevadas, superiores al $60 \%$ en determinados subgrupos ${ }^{3-5}$, este hecho no es ajeno a que la población penitenciaria española incluye una gran proporción de usuarios de drogas por vía parenteral (UDVP) y que España es el primer país de Europa en tasas de incidencia de Sida; suponiendo el colectivo de UDVP el 66,4\% de los casos de Sida declarados a diciembre de $1995^{6}$.

Las administraciones penitenciarias españolas ${ }^{7-8}$, siguiendo las recomendaciones del Consejo de Europa ${ }^{9}$ y la $\mathrm{OMS}^{10}$ han puesto en marcha desde finales de los años 80 programas de prevención y control de la infección por VIH en el medio carcelario.

El objetivo de este trabajo es conocer la evolución de la prevalencia de infección por VIH en el momento del ingreso en prisión y los factores asociados a la misma en este colectivo, lo que puede permitir una aproximación a la efectividad de las estrategias de reducción de riesgos y contribuir a mejorarlas.

\section{SUJETOS, MATERIAL Y MÉTODOS}

La población objeto del presente estudio fueron todas las personas que ingresaron en el Centro Penitenciario de León (CPL) entre el 1 de enero de 1991 y el 31 de diciembre de 1995. En aquellos pacientes que ingresaron en más de una ocasión durante el período en estudio sólo se consideró la primera de ellas. El CPL es una prisión provincial, con 300 reclusos de población estable y 600 ingresos al año, aproximadamente el 35\% UDVP o ex-UDVP, la gran mayoría procedente de los juzgados de la provincia y una minoría de otros centros penitenciarios (Madrid, Asturias y Galicia, fundamentalmente).
En el momento del ingreso se cumplimentó, por entrevista dirigida por personal sanitario previamente adiestrado, un cuestionario validado en estudios anteriores, donde se recogieron variables socio-demográficas (edad, sexo, estado civil, nivel socio-económico, nivel educativo), variables penitenciarias (número de ingresos en prisión, edad del primer ingreso, meses de permanencia en prisión, número de autolesiones) y factores de riesgo para la infección por VIH (uso de drogas por vía parenteral, patrón de uso, número de tatuajes). Una vez por semana se procedió a la recogida de una muestra de sangre para la determinación de infección por VIH, que se realizó, previo consentimiento informado preprueba y consejo médico postprueba, mediante técnica de ELISA (IMX HIV $1+2$ de Abbot ${ }^{\circledR}$, desde el inicio al 22.08.94 y de tercera generación a partir de dicha fecha) y confirmación mediante Inmunoblot (Innogenetics ${ }^{\circledR}$ ) en los casos positivos. En los casos en los que previamente se documentó infección por VIH fueron incluidos como infectados.

Los datos recogidos en el cuestionario fucron introducidos en una base de datos creada con el subprograma ENTER del programa informático EPIINFO versión 5 y tratados con el subprograma ANALISYS del mismo ${ }^{11}$. Para el análisis estadístico se utilizó la prueba de ji-cuadrado con la corrección de Yates para las variables cualitativas; para las variables cuantitativas se utilizó el análisis de la varianza en el caso de normalidad demostrada con el test de Bartlett; de no existir homogeneidad se utilizó la prueba no paramétrica de Kruskal-Wallis. El análisis de la tendencia y estratificado se llevó a cabo con el programa STATCALT para tendencias del programa EPIINFO versión 5 (Ji-cuadrado extensión de Mantel). Para el análisis de regresión logística se utilizó el programa EGRET y el método de máxima verosimilitud y se incluyeron en él todas las variables que a nivel univariado se asociaban a la infección VIH con una $\mathrm{P}<0,1^{12}$. 


\section{RESULTADOS}

Durante el período en estudio 2.252 personas ingresaron al menos una vez en el centro penitenciario y fueron incluidas en el mismo. Las características sociodemográficas de este colectivo pueden observarse en la tabla 1.

Se trataba de una población joven, con una edad media de 30,1 $\pm 9,3$ años (mediana=28; P25-P75=24-34); mayoritariamente masculina $(89,4 \%)$ y de raza blanca $(88,1 \%)$. Más de la mitad de la población carecía de cualificación laboral $(56,3 \%)$ y un porcentaje similar no había obtenido el graduado escolar o estudios equivalentes $(56,1 \%)$. El $60 \%$ de los entrevistados eran solteros. El $55,1 \%$ de la población presentaba al menos un tatuaje y aproximadamente el $17 \%$ de los ingresos estudiados refirió antecedentes de lesiones autoinfringidas. El 35,4\% de los estudiados se declaró UDVP, el 2,6\% de forma esporádica en el último año, el $69,7 \%$ de forma habitual (diaria o casi diaria) en el último año y el $27,6 \%$ ser ex-consumidores (un año o más sin consumir por vía parenteral). De los 796 UDVP o Ex-UDVP, más de la mitad $(54,7 \%)$ manifestaron compartir o haber compartido material de inyección.

La proporción ingresos UDVP descendió de forma mantenida y significativa $(\mathrm{P}<0,00001)$ durante los años de estudio incluso estratificando según existieran o no antecedente de permanencias previas en prisión. (figura 1).

Algo menos de la mitad de la población no había estado nunca en prisión $(43,7 \%)$, del $56,3 \%$ restante la media de ingresos en prisión fue de 4,4 $\pm 5,1$ (mediana=3; P25$\mathrm{P} 75=2-5$ ); el primer ingreso en prisión se produjo como media a una edad de $23,2 \pm 8,0$ años (Mediana $=21, P 25-P 75=17-27)$ y el tiempo total de permanencia en prisión previo al ingreso objeto de estudio fue de $31,8 \pm 41,3$ meses (Mediana $=14$; P25P75=3-48), en más de la mitad $(56,8 \%)$ fue igual o superior a un año.
Tabla 1

Características de la población incluida inicialmente en el estudio

\begin{tabular}{|c|c|c|}
\hline Variables & $N$ & $\%$ \\
\hline \multicolumn{3}{|l|}{ Año } \\
\hline 1991 & 648 & 28.8 \\
\hline 1992 & 510 & 22,6 \\
\hline 1993 & 464 & 20,6 \\
\hline 1994 & 325 & 14.4 \\
\hline 1995 & 305 & 13.5 \\
\hline \multicolumn{3}{|l|}{ Sexo } \\
\hline Hombre & 2.013 & 89.4 \\
\hline Mujer & 239 & 10,6 \\
\hline \multicolumn{3}{|l|}{ Grupos de edad } \\
\hline $16-24$ & 651 & 28,9 \\
\hline $25-34$ & 1.039 & 46.2 \\
\hline $35-44$ & 388 & 17.2 \\
\hline$>44$ & 173 & 7,7 \\
\hline \multicolumn{3}{|l|}{ Etnia } \\
\hline Blanco & 1.982 & 88.1 \\
\hline Gitano & 219 & 9.7 \\
\hline Otros & 50 & 2,2 \\
\hline \multicolumn{3}{|l|}{ Cualificación laboral } \\
\hline No & 1.257 & 56,3 \\
\hline Sí & 974 & 43,7 \\
\hline \multicolumn{3}{|l|}{ Graduado escolar } \\
\hline No & 1.219 & 56.1 \\
\hline Sí & 955 & 43,9 \\
\hline \multicolumn{3}{|l|}{ I'atuajes } \\
\hline Sí & 986 & 44.9 \\
\hline No & 1.208 & 55,1 \\
\hline \multicolumn{3}{|c|}{ Antecedentes de autolesiones } \\
\hline Sí & 356 & 16.6 \\
\hline No & 1.788 & 83,4 \\
\hline \multicolumn{3}{|l|}{ UDVP } \\
\hline Sí & 796 & 35.4 \\
\hline No & 1.454 & 64.6 \\
\hline \multicolumn{3}{|l|}{ Compatir jeringuillas } \\
\hline Sí & 428 & 54.7 \\
\hline No & 355 & 45,3 \\
\hline \multicolumn{3}{|l|}{ Tiempo en prisión } \\
\hline Primer ingreso & 984 & 43.7 \\
\hline Menos de un año & 555 & 24.6 \\
\hline Un año o más & 713 & 31.7 \\
\hline
\end{tabular}

De los 2252 sujetos objeto de estudio pudo conocerse su estado respecto a la infección en $1663(73,8 \%)$. En 40 casos (1\%) se debió a negativa del paciente a la extracción 
Figura 1

Distribución del UDVP en población reclusa según antecedentes de estancias previas en prisión. Años 1991-95

PREVALENCIA \%

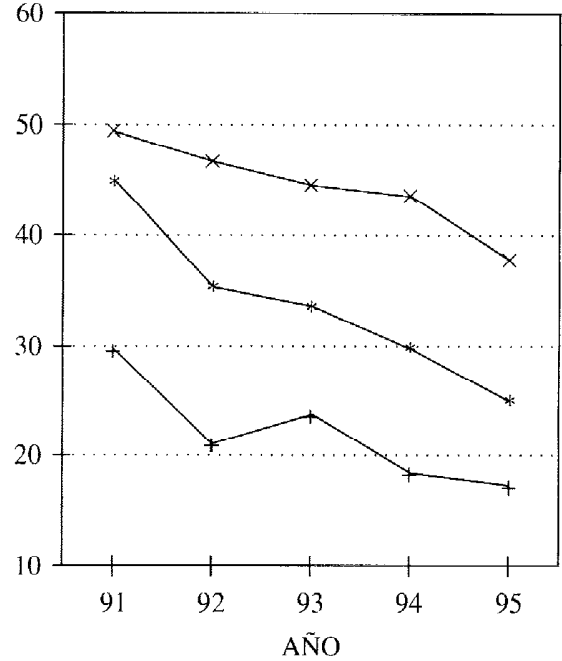

sanguínea y en los 573 casos restantes a que no permanecieron en el centro el tiempo necesario para la toma de muestras. El análisis de las pérdidas no objetivó diferencias significativas más que para variables asociadas al tiempo de permanencia en prisión, siendo con más frecuencia las pérdidas no UDVP y personas que ingresaron por vez primera en prisión. La tendencia de las pérdidas mostró que estas fueron más frecuentes en los últimos años del estudio que en los primeros $(\mathrm{P}<0,001)$.

De las 1663 personas estudiadas fueron clasificadas como infectadas 322 lo que supuso una prevalencia de infección VIH del $\mathbf{1 9 , 4 \%}$ (I.C. $95 \%=17,5 \%-21,3 \%$ ) cuya distribución según las variables estudiadas puede observarse en la tabla 2.

La prevalencia de infección fue superior en las mujeres $(26,0 \%)$ que en los hombres $(18,6 \%)(\mathrm{O} . \mathrm{R}=1,54)$ y el grupo de edad de 25 a 34 años presentó la mayor proporción de infectados $(29,1 \%) \quad(\mathrm{P}<0,000001)$. La media de edad de los infectados por VIH fue de 28,4 $\pm 5,0$ años (Mediana=28 años; P25P75=25-31 años) y de 30,3 $\pm 9,7$ años en los $\times$ Con antecedentes $\star+$ Sin antecedentes $\star \star *$ Total $\star \star \star$

$\star \star \star$ Global: $\mathrm{P}<0,00001$; Análisis estratilicado: $\mathrm{P}<0,0004$

$\star \mathrm{P}=0,014 \star \star \mathrm{P}=0,008$ no infectados (Mediana $=28$ años; $\mathrm{P} 25$ $\mathrm{P} 75=23-35$ años $)(\mathrm{P}=0,33)$. La distribución de la infección fue más elevada en las personas de raza blanca $(17 \%)$ que en las de raza gitana $(10,0 \%)$ o en el grupo otros $(7,9 \%)$ que incluía fundamentalmente personas procedentes de países del Magreb y centroafricanos. La infección era ligeramente más elevada, aunque no significativamente, en los ingresos no cualificados laboralmente que en los cualificados $(20,0 \%$ vs $18,4 \%$; $\mathrm{P}=0,43$ ); sí se observaron diferencias significativas $(\mathrm{P}=0,044)$ entre aquellos internos que habían obtenido el graduado escolar o equivalente $(21,6 \%)$ respecto a quienes no habían obtenido dicha cualificación académica $(17,5 \%)(O . R .=1,16)$. La infección se mostró también asociada al estado civil, siendo mayor en los solteros $(22,8 \%)$ que en los casados $(14,3 \%)$ o en los separados-divorciados-viudos $(18,8 \%)$. También los tatuados y los que presentaron antecedentes de autolesiones presentaban niveles de infección VIH significativamente más elevados que sus contrarios $(29,9 \%$ vs $9,4 \%$; y $60,5 \%$ vs $14,3 \%$ ). La variable que presentó mayor asociación con la infección VIH fue el antecedente de uso de drogas por vía parenteral 
Tabla 2

Distribución de la infección por VIH según diversas variables

\begin{tabular}{|c|c|c|c|c|c|}
\hline Variables & Número & $V I H(+)$ & $\%$ & $O . R$ & I.C. $95 \%$ \\
\hline \multicolumn{6}{|l|}{ Sexo } \\
\hline Hombre & 1.494 & 278 & 18,6 & 1,54 & $1,04-2,27$ \\
\hline Mujer & 169 & 44 & 26.0 & 1 & \\
\hline \multicolumn{6}{|l|}{ Grupos de edad } \\
\hline $16-24$ & 488 & 63 & 12,9 & 8,45 & $2,18-72,22$ \\
\hline $25-34$ & 776 & 226 & 29,1 & 23,42 & $6,23-197.07$ \\
\hline $35-44$ & 282 & 30 & 10,6 & 6,79 & $1,67-59,40$ \\
\hline$>44$ & 116 & 2 & 1,7 & 1 & \\
\hline \multicolumn{6}{|l|}{ Etnia } \\
\hline Blanco & 1.45 .5 & 302 & 20,9 & 2.36 & $1,40-4,22$ \\
\hline Gitano & 170 & 17 & 10,0 & 1 & \\
\hline Otros & 38 & 3 & 7,9 & 0,77 & $0,14-2,89$ \\
\hline \multicolumn{6}{|l|}{ Cualificación laboral } \\
\hline Sí & 708 & 130 & 18.4 & 1,11 & $0,86-1,+4$ \\
\hline No & 944 & 189 & 20,0 & 1 & \\
\hline \multicolumn{6}{|l|}{ Graduado escolar } \\
\hline Sí & 680 & 147 & 21,6 & 1,30 & $1,00-1,69$ \\
\hline No & 927 & 162 & 17,5 & 1 & \\
\hline \multicolumn{6}{|l|}{ Estado civil } \\
\hline Soltero & 92.3 & 210 & 22.8 & 1,77 & $1,32-2.38$ \\
\hline Casado & 55,3 & 79 & 14.3 & 1 & \\
\hline Otros & 170 & 32 & 18,8 & 1.39 & $0.85-2,23$ \\
\hline \multicolumn{6}{|l|}{ Tatuajes } \\
\hline Sí & 778 & 233 & 29,9 & 4.11 & $3.08-5.50$ \\
\hline No & $851)$ & 80 & 9,4 & 1 & \\
\hline \multicolumn{6}{|l|}{ Autolesión } \\
\hline Sí & $26^{4}$ & 114 & 42,4 & 4,41 & $3,26-5,95$ \\
\hline No & 1.321 & 189 & 14.5 & 1 & \\
\hline \multicolumn{6}{|l|}{ UDVP } \\
\hline Sí & 646 & 299 & 46,3 & 37.24 & $23,80-60,57$ \\
\hline No & 1.017 & 23 & 2.3 & 1 & \\
\hline \multicolumn{6}{|l|}{ Compartir jeringuillas } \\
\hline Sí & 371 & 228 & 61,5 & 4.58 & $3.20-6.57$ \\
\hline No & 275 & 71 & 25.8 & 1 & \\
\hline \multicolumn{6}{|l|}{ Tiempo en prisión previo } \\
\hline Primer ingreso & 694 & 51 & 7,3 & 1 & \\
\hline Menos de 12 meses & 451 & 78 & 17,3 & 2,64 & $1.78-3,92$ \\
\hline 12 meses o más & 518 & 193 & 37.3 & 7.49 & $5,30-10.69$ \\
\hline
\end{tabular}

(O.R. $=37,24)$ y entre estos aquellos que manifestaron haber compartido jeringuillas presentaban las prevalencias de infección más elevadas $(61,5 \%)$.

La infección VIH fue más elevada en aquellos que tenían antecedentes previos de ingreso en prisión que en aquellos que ingresaron por vez primera en el momento del estudio $(28,0 \%$ vs $7,3 \%$; O.R. $=4,90)$; y en aquellos con antecedentes previos de ingreso los que habían permanecido en ella un año o más presentaban mayores niveles de infección que quienes habían permanecido menos 
de un año $(37,3 \%$ vs $17,3 \%$; O.R.=2,84). Entre aquellos con antecedentes de ingresos previos en prisión, los infectados por VIH presentaban un mayor número de ingresos que los no infectados (Media $=6 \pm 5$, mediana $=4, P 25-P 75=3-8$; versus; Media $=4 \pm 5$, mediana $=2$, P25-P $75=2-4, \quad \mathrm{P}<0,00001$ ); una mayor precocidad en el primer ingreso en prisión (Media $=20 \pm 5$ años, mediana $=18$, P25-P75 $=16-22$; versus; Media $=24 \pm 8$ años, mediana $=22 ; \mathrm{P} 25-\mathrm{P} 75=18-28, \mathrm{P}<0,00001$ ) $\mathrm{y}$ un mayor número de meses previos en prisión (Media $=42 \pm 41$, mediana $=30$, P25 $\mathrm{P} 75=8-65$; versus; Media $=26 \pm 39$, media$\mathrm{na}=9 ; \mathrm{P} 25-\mathrm{P} 75=2-32, \mathrm{P}<0,00001$ ).

La prevalencia de infección VIH descendió de forma significativa $(\mathrm{P}=0,00001)$ durante los años en estudio, desde el $24,1 \%$ del año 1991 al $11,2 \%$ en el año 1995 $(\mathrm{O} . \mathrm{R} .=0,40)$. En el análisis estratificado de la tendencia de la infección en función de la existencia o no de permanencias previas en prisión, se mantuvo el descenso en los niveles de infección pero rozando la significación estadística $(\mathrm{P}=0,064 ; \mathrm{O} . \mathrm{R} .=0,63)$. En aquellos ingresos sin antecedentes de estancias previas en prisión el descenso fue acu- sado (del $12,4 \%$ en 1991 al 3,2 \% en 1995) y las diferencias observadas se mostraron estadísticamente significativas $(\mathrm{P}=0,008$, $\mathrm{O} . \mathrm{R}=0,23$ ). Sin embargo, en los estudiados con antecedentes previos de ingreso o estanciaen prisión no se objetivaron cambios significativos $(\mathrm{P}=0,44)$ (figura 2$)$.

El análisis de la tendencia de la infección por VIH estratificada según la variable UDVP no mostró cambios significativos ni en la tendencia global ni para ninguna de las categorías. (figura 3 ).

El análisis de regresión logística que mejor explicó la distribución de la infección VIH incluyó las variables: año, sexo, grupos de edad, etnia, tatuajes, autolesiones, UDVP y tiempo previo en prisión. Siendo la infección más frecuente durante el año 1992, en las mujeres, en aquellos que presentaban tatuajes y antecedentes de autolesiones, y en aquellos que manifestaban antecedentes de estancia en prisión, fundamentalmente en aquellos con permanencia durante un año o más. La etnia gitana se asociaba con una menor probabilidad de infección VIH en relación a los otros grupos (tabla 3).

Figura 2

Distribución de la infección por VIH en población reclusa según antecedentes de estancias previas en prisión. Años 1991-95 PREVALENCIA \%

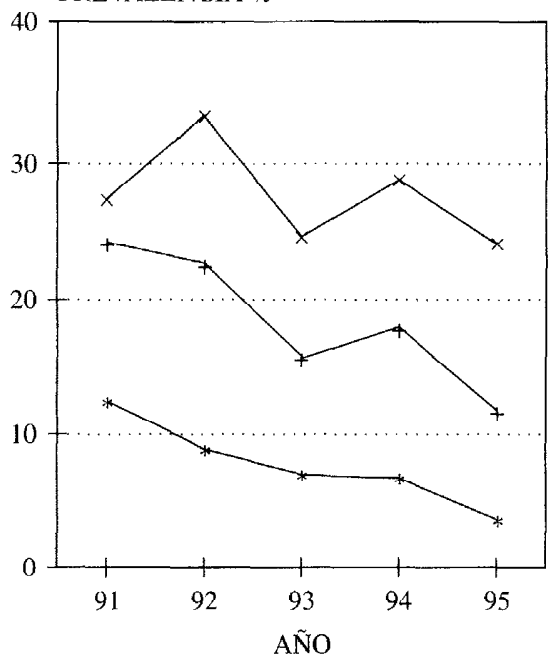

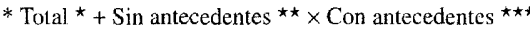

* Global: $P<0,00001$; Análisis estratificado: $P<0.064$

${ }^{\star \star} \mathrm{P}=0,008{ }^{\star \star} \mathrm{P}=0,44$ 
Figura 3

Distribución de la infección por VIH en población reclusa según antecedentes de UDVP. Años 1991-95

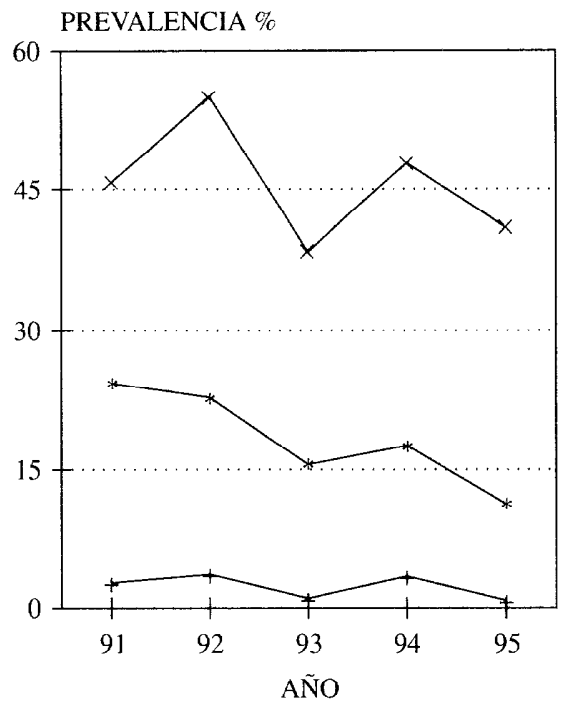

DISCUSIÓN

La prevalencia de infección por VIH observada es muy elevada, entre 30 y 40 veces mayor que la estimada para población general en nuestro país $(3-4 \text { por mil })^{13}$. La mayoría de los trabajos publicados sobre población reclusa de otros países refieren prevalencias inferiores al dos por ciento ${ }^{14-26}$, algunos entre el $2 \%$ y el $5 \%{ }^{27-31}$, pocos superiores al 5\% ${ }^{32-39}$; y sólo la población reclusa de Nueva York presenta cifras similares a las de este trabajo ${ }^{40,41}$.

La prevalencia encontrada, sin embargo, es inferior a la reportada por la mayoría de los estudios en población reclusa española que oscilan entre el $24,4 \%$ y el $65,2 \% \%^{35,42,13}$. Las diferencias observadas pueden deberse al diferente reclutamiento de la población a estudio (seleccionada y población estable), a divergencias en la extensión de las prácticas de riesgo, en la prevalencia de infección en el medio de origen ${ }^{5}$ y al tiempo transcurrido entre los trabajos. Nuestros resultados son similares a otras publicaciones más mo-

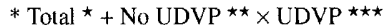

$\star$ Global: $P<0,00001 ;$ Análisis estratificado: $P<0,16$

$\star \star * P=0,20{ }^{\star \star \star} \mathrm{P}=0,31$
}

dernas y que estudian población al ingreso en prisión $(28,4-15,8 \%)^{44-46}$.

La infección por VIH suele ser más prevalente en los grupos étnicos minoritarios ${ }^{47}$. En nuestro caso, la principal minoría étnica de nuestro país, los gitanos, se encuentran menos infectados que los blancos. Este hecho esta en contradicción con lo encontrado en otro trabajo sobre población reclusa española ${ }^{3}$. Una posible explicación a este hecho, dado el tiempo transcurrido entre ambos estudios, tal vez sea una mejor y mas rápida aceptación en esta etnia, de prácticas más seguras; aunque se trata de una minoría marginada social y económicamente mantienen fuertes lazos familiares y reglas sociales y tradiciones muy acendradas que pueden haber favorecido estos posibles cambios de hábitos ${ }^{47}$.

El mayor nivel de infección en la población reclusa femenina que en la masculina ha sido puesto de manifiesto en otros trabajos ${ }^{15,18,27,29,31,36,45}$. El mayor riesgo de la mujer en las prácticas heterosexuales desprotegidas, una mayor exposición a VIH por no ser 
Tabla 3

Análisis de regresión logística en población total

\begin{tabular}{|c|c|c|c|c|}
\hline Variables & Coeficiente & Odds Rutio & I.C. $95 \%$ & Vulor de $P$ \\
\hline \multicolumn{5}{|l|}{ Año } \\
\hline 1992 & 0,5989 & 1,820 & $1,18-2,82$ & 0,007 \\
\hline 1993 & -0.0401 & 0.96 & $0,60-1,54$ & 0.867 \\
\hline 1994 & 0,4540 & 1,58 & $0,89-2.80$ & 0,122 \\
\hline 1995 & 0.1614 & 1,18 & $0,62-2,22$ & 0.621 \\
\hline \multicolumn{5}{|l|}{ Sexo } \\
\hline Mujer & $-5,405$ & 2,15 & $1,26-3,66$ & $<0,001$ \\
\hline \multicolumn{5}{|l|}{ Grupos de edad } \\
\hline 25-34 Años & 0,9143 & 2,50 & $1,68-3,70$ & $<0,001$ \\
\hline 35-44 Años & 0.6332 & 1,88 & $1.01-3.52$ & 0,047 \\
\hline >44 Años & 0,2341 & 1.26 & $0.27-5.89$ & 0,766 \\
\hline \multicolumn{5}{|l|}{ Etnia } \\
\hline Blancos & $-0,0755$ & 0,93 & $0,21-4,14$ & 0,921 \\
\hline Gitanos & $-1,043$ & 0,35 & $0,18-0,67$ & 0.002 \\
\hline \multicolumn{5}{|l|}{ Tatuajes } \\
\hline Sí & 0,4809 & 1.62 & $1.11-2.35$ & 0,011 \\
\hline \multicolumn{5}{|l|}{ Autolesiones } \\
\hline Sí & 0.3846 & 1.47 & $1.01-2.14$ & 0,044 \\
\hline \multicolumn{5}{|l|}{ UDVP } \\
\hline Sí & 3,245 & 25,66 & $15.63-42,15$ & $<0,001$ \\
\hline \multicolumn{5}{|l|}{ Meses prisión } \\
\hline <de 12 meses & 0,4130 & 1,51 & $0,95-2,40$ & 0.081 \\
\hline$>$ de 11 meses & 1,235 & 3,44 & $2,18-5,43$ & $<0,001$ \\
\hline
\end{tabular}

infrecuente que su pareja esté infectada por él y la práctica de la prostitución como medio de vida o de financiación de su toxicomanía y/o la de su pareja afectiva pueden explicar estos hallazgos ${ }^{30,45,48}$.

La asociación de los tatuajes con la infección $\mathrm{V} I \mathrm{H}$ ha sido reportada en población reclusa ${ }^{4.7 .49}$. Otros autores han observado que esta asociación desaparecía al estratificar por UDVP ${ }^{3}$ o se atenuaba mucho su relación con la infección ${ }^{5}$. Aún estando reconocida esta práctica como vía de transmisión de VIH, en nuestro caso tal vez traduzca características diferenciales de la población toxicómana tatuada versus la no tatuada: menor capacidad de percepción del riesgo, mayores niveles de asocialidad o marginalidad y prácticas de riesgo más frecuentes y extendidas ${ }^{5}$. Este razonamiento puede también explicar el mayor riesgo de infección en aquellos internos que refirieron prácticas autolesivas.

La asociación del tiempo de estancia en prisión con la infección VIH ha sido reportada por algunos autores ${ }^{3,5}$ y varios trabajos recogen como esta infección es mayor en los UDVP que han ingresado en prisión que en los que no ${ }^{47,50,51}$. Otros autores lo asocian al mayor tiempo de consumo y no al tiempo de encarcelamiento ${ }^{52}$, otros no han observado transmisión de la infección por VIH en prisión ${ }^{45.53}$. Sin embargo la evidencia de la transmisión de ésta en el interior de las prisiones ha sido documentada en diversos paí$\operatorname{ses}^{22,27,30,54-56}$. Un mayor tiempo de permanencia en prisión supone una mayor probabilidad de consumir en ella, la penuria de material estéril de inyección facilita el compartirlo y el control funcionarial el consumo en condiciones antihigiénicas; lo que unido 
a las elevadas prevalencias del medio supone una situación de altísimo riesgo para contraer la infección. La asociación del número de ingresos en prisión (primario versus reincidente) puede traducir características diferenciales de los UDVP reincidentes y/o situaciones de alto riesgo de consumo con prácticas de riesgo por los síndromes de abstinencia sufridos al ingreso en prisión.

El uso de drogas por vía parenteral es la variable que presenta una mayor asociación con la infección, tal y como se ha observado en la población general española y en la casi totalidad de los trabajos publicados sobre población reclusa a nivel internacional. De los 23 casos no catalogados como UDVP, confrontando nuestra base de datos con otros registros (ficheros de asistencia social, registro de casos de SIDA), se constató como en 18 casos se trataba de UDVP que no lo reconocieron a los servicios médicos, en un caso la transmisión de la infección fue probablemente por prácticas homosexuales y en cuatro por prácticas heterosexuales. El compartir el material de inyección es probablemente el principal factor de riesgo en la transmisión de la infección por VIH en los UDVP ${ }^{57}$ y así se observa en nuestro trabajo.

La mayor infección VIH en los grupos de edad comprendidos entre los 25 y los 44 años puede traducir una mayor infección por un mayor tiempo de exposición asociado a la edad, y la escasa afectación del grupo de edad superior 44 años la escasa representación en ese grupo de edad del colectivo UDVP.

No encontramos ninguna justificación al hecho que se haya observado una mayor proporción de infectados en aquellos que ingresaron en el año 1992.

El descenso observado, mantenido y significativo, en la prevalencia de infección global durante los años en estudio podía haberse debido a un sesgo de selección dado que los reingresos durante el período en estudio solo eran considerados en su primer ingreso, en este colectivo es habitual una sobrerrepresentación de UDVP y por tanto de infección VIH. El estudio estratificado de la tendencia en función de los antecedentes de estancia previa en prisión viene a equilibrar de alguna manera dicho sesgo y permitió mostrar como se mantiene el descenso observado, si bien de una forma más moderada, y muy cerca pero sin alcanzar ya la significación estadística. Una de las posibles explicaciones a este hecho puede radicar en el diferente comportamiento de los estudiados según sus antecedentes penitenciarios; así, se objetivó una disminución de la infección importante y significativa en aquellos que en el momento del estudio ingresaron por vez primera en su vida en prisión, y no se observó diferencia en la tendencia de la infección en aquellos sujetos que con anterioridad habían estado en la cárcel. Este hecho puede obedecer bien al éxito de los programas de prevención llevados a cabo en la calle y al relativo fracaso de los programas en aquellos que han ingresado en prisión. También a factores demográficos diferenciales entre los primarios y los reincidentes, tales como una menor edad o un menor tiempo de consumo o en el uso de otras vías de consumo; y que el paso del tiempo puede llevar a igualar las prevalencias por modificaciones en los hábitos de consumo de drogas.

El análisis de la evolución de la infección según UDVP puede abundar en esta idea toda vez que los UDVP se mantienen igual de infectados a lo largo de los años en estudio. La tendencia de la proporción de UDVP en los años de estudio también puede dar claves sobre lo sucedido; una menor proporción de UDVP al ingreso en prisión puede deberse efectivamente a una menor proporción o bien a un problema del método de estudio y muchos de los primarios que en ese momento no son UDVP en el momento de otro ingreso en prisión, como reincidentes sí lo sean. Sin embargo, el hecho de haber observado incluso una modificación a la baja de la proporción de UDVP entre los reincidentes puede significar efectivamente un menor uso de la vía parenteral en este colectivo. 
Tal vez la explicación del descenso observado en la infección VIH se deba en gran medida a una disminución en la proporción de UDVP que ingresan en prisión, sin embargo, el mantenimiento de los niveles de infección en los UDVP, tanto en primarios como en reincidentes, puede explicar que la prevalencia en la población global no haya disminuido tanto como se hubiera esperado o sería deseable. Aunque los UDVP han reducido la frecuencia en las prácticas de riesgo se mantiene el porcentaje de UDVP que en alguna ocasión comparten jeringuillas (más del 60\%). Si en el momento de decidir el consumo no se cuenta con material estéril de inyección a mano este se va a compartir; lo extendido de la infección hacen de esta práctica excepcional una ocasión de muy alto riesgo para el contagio, más elevada aun en el caso de las prisiones $^{47,48}$

Como conclusión constatamos el mantenimiento de las elevadas prevalencias de infección por VIH en este colectivo diez años después de conocer la gravedad de la situación. Se ha preconizado la abstinencia como objetivo universal e irrenunciable en el tratamiento de toxicómanos y esta costando mucho considerar otros objetivos intermedios tales como la reducción del daño ${ }^{58}$ cuando se han demostrado compatible estos programas con programas libres de drogas $^{59}$. Como resultado se ha tardado mucho y no se han ofertado de manera efectiva programas de mantenimiento con metadona. El mantenimiento, a menor escala del uso de la vía parenteral compartiendo jeringuillas, tanto dentro como fuera de prisión, dadas las altas prevalencias del medio, hace necesario plantearse la entrega de material de inyección estéril. Esta medida plantea muchos inconvenientes para su puesta en práctica en el medio penitenciario, no tenemos la respuesta y sí muchas preguntas. Tal vez la constitución de un grupo de trabajo y tres o cuatro programas pilotos evaluables a corto plazo podrían ofrecer luz sobre estos aspectos.

\section{BIBLIOGRAFÍA}

1. Harding TW. AIDS in prison. Lancet 1987; $2: 1260-3$.

2. Hammett TM. Update 1989: AIDS in Correctional Facilities. Washington, DC: National Institute of Justice; 1990.

3. Martín V, Bayas JM, Laliga A, Pumarola T, Vidal J, Jimnez de Anta M'I, Salleras L. Seroepidemiology of HIV-1 infection in a Catalonian penitentiary. AIDS 1990; 4:1023-1026.

4. Estébanez P, Colomo C, Zunzunegui MV, Rua M, Pérez M, Ortiz C, et al. Cárceles y SIDA . Factores de riesgo de infección por el VIH en las cárceles de Madrid. Gac Sanit 1990; 4:100-5.

5. Bayas .IM, Martín V, Vidal J, Pumarola T, Jiménez de Anta MT, Salleras L. Infcción por el virus de la inmunodeficiencia humana tipo 1 en la población penitenciaria de Cataluña. Med Clin (Barc) 1994; 102:209-212.

6. Registro Nacional de casos de SID $\Lambda$ en España. Informe Trimestral 4/95. Centro Nacional de Epidemiología, Instituto de Salud Carlos III. Madrid; 1996.

7. Martín M. Programa de Prevención y Control de Enfermedades Transmisibles en Instituciones Penitenciarias. Rev Estudios Penitenciarios. Monográfico de Sanidad Penitenciaria Extra-1-1990: 51-67.

8. Ballester J. Problemática en prisiones. II Reunión sobre el SIDA. Publicación oficial de la SEISIDA 1994; 3:137-139.

9. Programa Especial sobre el SIDA. Declaración de la Reunión Consultiva sobre prevención y lucha contra el SIDA en las cárceles. Ginebra: OMS; 1987.

10. Global Programme on AIDS. WHO guidelines on HIV infection and AIDS in prisons. Ginebra: OMS 1993; WHO/GPA/DIR/93.3.

11. Dean AD, Dean JA, Burton AH, Dicker RC. Epi Info, version 5: A word processing, database, and statistics program for epidemiology on microcomputers. Stone Mountain, Georgia: USD Incorporated; 1990

12. Epidemiological Graphics, Estimation and Testing Package (EGRET). Seattle, Washington: Statistics and Epidemiology Research Corporation; 1990.

13. Rey $\mathrm{R}$, Musina V, Casal M, Caylà JA, De March $\mathrm{P}$, Morcno S, et al. Situación actual de la tuberculosis en España. Una perspectiva sanitaria en precario respecto a los países desarrollados. Med Clín (Barc) 1995; 105:703-707. 
14. Sweeney P, Lindegren ML, Buehler JW, Onorato IM, Janssen RS. Teenagers at rish of human immunodeficiency virus type 1 infection. results from seroprevalence surveys in the United States. Arch Pediatr Adolesc Med 1995; 149(5):521-8.

15. Patel KK, Hutchinson C, Sienko DG. Sentinel surveillance of HIV infection among new inmates and implications for policies of corrections facilities. Public Health Rep 1990; 105(5):510-4.

16. Hoxie NJ. Vergeront JM, Frisby HR, Pfister JR, Golubjatnikov R, Davis JP. HIV seroprevalence and the acceptance of voluntary HIV testing among newly incarcerated male prison inmates in Wisconsin. Am J Public Health 1990; 80(9):1129-31.

17. Calzavara LM, Major C, Myers T, Schlossberg J, Millson $\mathrm{M}$, Wallace $\mathrm{E}$, et al. reducing volunteer bias: using left-over specimens rates of HIV infection among inmates in Ontario, Canada. AIDS 1995; $9(6): 613-7$.

18. Rothon DA, Mathias RG, Schechter MT. Prevalence of HIV infection in provincial prisons in British Columbia (see comments). Can Med Assoc J 1995; 152(6):781-7.

19. Crofts N, Stewart T, Hearne P, Ping XY, Breshkin AM, Locarnini SA. Spread of bloodborne virus among Australian prison entrants. BMJ 1995; 310 (6975): 285-288.

20. Gaughwin MD, Douglas RM, Liew C, Davies L, Mylvaganam A, Treffke $H$, et al. HIV prevalence and risk behaviours for HIV transmission in South Australian prison. AIDS 1991; 5(7):84551.

21. Guereña Burgueño F, Benenson AS, Sepulveda Amor J. HIV-1 prevalence in selected Tijuana sub-populations. Am J Public Health 1991; 81(5):623-5.

22. Gore SM, Bird AG, Burns SM, Goldberg DJ, Ross AJ, Macgegor J. Drug injection and HIV prevalence in inmates of glenochil prison (see comments). BMJ 1995; 310(6975):293-6.

23. Pont J, Strutz H, Kahl W, Salzner G. HIV epidemiology and risk behavoir promoting HIV transmission in Austrian prisons. Eur $\mathbf{J}$ Epidemiol 1994: 10(3):285-9.

24. Ousseini H. Seroprevalence de l'infection au virus de l'immunodeficience humaine (VIH) a la prison centrale de Niamey (Niger). Bull Soc Pathol Exot 1994; 87(3):90.

25. Vaz RG, Gloyd S, Folgosa E, Kreiss J. Syphilis and HIV infection among prisoners in Maputo, Mozambique. Int J STD AIDS 1995: 6(1):42-46.
26. Dasananjali T. The prevalence of HIV infection among mentally ill offenders in Thailand. J Med Assoc Thai $1994 ; 77(5): 257-60$.

27. Bird AG, Gore SM, Jolliffe DW, Burns SM. Anonymous HIV surveillance in Saughton Prison, Edinburgh. AIDS 1992; 6: 725-733.

28. Papaevangelou G, Roumeliotou A, Stergioy G, Nesordiou A, Trichopoulou E, Kallinikos G, et al. HIV infection in Greek intravenous drug users. Eur J Epidemiol 1991; 7(1):88-90.

29. Singleton JA, Perkins CI, Trachtenberg AI, Hughes MJ, Kizer KW, Ascher M. HIV antibody seroprevalence among prisoners entering the California correctional system. West J Med 1990: 153(4): 394-9.

30. Gellert GA, Maxwell RM, Higgins KV, Pendergast T, Wilker N. HIV infection in the Women's Jail Orange County, California, 1985 through 1991. Am J Public Health 1993; 83(10):1454-6.

31. Vlahov D, Brewer TF, Castro KG, Narkunas JP, Salive ME, Ullrich J, et al. Prevalence of antibody to HIV-1 among entrants to US correctional facilities. JAMA 1991; 265(9):1129-32.

32. Hankins CA, Gendron S, Handley MA, Richard C, Tung MT, O'Shaughnessy M. HIV infection among women in prison an assesment of risk factors using a nonnominal methodology. Am J Public Health $1994 ; 84(10): 1637-40$.

33. Rotily M, Galinier-Pujol A, Obadia Y, Moatti JP, Toubiana P, Vernay-Vaisse C, et al. HIV testing, HIV infection and associatcd risk factors among inmates in south-eastern French prisons. AIDS $1994 ; 8(9): 1341-4$

34. Vlahov D, Muñoz A, Brewer $\mathrm{f}$, Taylor E, Canner C. Polk BF. Sasonal and annual variation of antibody to HIV-I among male inmates entering Maryland prisons:update. AIDS $1990 ; 4(4): 345-50$.

35. Vlahov D, Brewer TF, Castro KG, Narkunas JP, Salive ME, Ullrich J, et al. Prevalence of antibody to HIV-1 among entrants to US correctional facilities. JAMA 1991; 265(9):1129-32.

36. Behrendt C, Kendig N, Dambita C, Horman J, Lawlor J, Vlahov D. Voluntary testing for human immunodeficiency virus (HIV) in a prison population with a high prevalence of HIV. Am J Epidemiol 1994; 139(9):918-26.

37. Quarto M, Germinario C, Troiano T, Fontana A, Barbuti S. Tossicodipendenza ed infezione da HIV: valutazione epidemiologica in una popolazione carceraria. Riv Eur Sci Med Faracol 1990; $12(2): 119-25$. 
38. Ippolito G. Gli ammalati di AIDS nelle carceri italiane: un problema scottante di non facile soluzione. Recenti Prog Med 1993; 84(1):57-63.

39. Kebede Y, Pickering J, McDonald JC, Wotton K, Zewde D. HIV infection in an Ethiopian prison. Am J Public Health 1991; 81(5):625-7.

40. Weisfuse IB, Greenberg BL, Back SD, Makki HA, Thomas P, Rooney W, et al. HIV-1 infection among New York inmates. AIDS 1991; 5(9): 1133-8.

41. Smith PF, Mikl J, Truman BI, Lessner L, Lehman JS, Stevens RW, et al. HIV infection among women entering the New York State correctional system. Am J Public Health 1991; 81 Suppl:3540 .

42. Eiros JM, Ortiz de Lejarazu R, Orduña A, Castrodeza J, Perlado E, Rodríguez A. Infección por virus del SIDA en grupos de interés sanitario. Valladolid: Diputación Provincial de Valladolid; 1990.

43. Caballero J, Rojas O, García A, García A. Infección por VIH y factores de reisgo asociados en la prisión de Guadalajara. Libro de Ponencias y Comunicaciones. I Congreso Nacional de Sanidad Penitenciaria. León; 1993. p. 344.

44. Diez M. Situación de la infección por VIH en las Instituciones Penitenciarias. Libro de Ponencias y Comunicaciones. I Congreso Nacional de Sanidad Penitenciaria. León; 1993. p. 179-84.

45. Anónimo. Situación socio-sanitaria de la población que ha ingresado en prisiones en 1993. Madrid: Ministerio de Justicia; 1994.

46. Pallás JR, Ruiz JC. Infecciones víricas transmitidas por via parenteral. Prevalencia en el C.P. de Santander.Libro de Ponencias y Comunicaciones. I Congreso Nacional de Sanidad Penitenciaria. León; 1993. p. 259.

47. Des Jarlais DC, Friedman SR, Choopanya K, Vanichseni S, Ward TP. International epidemiology of HIV and AIDS among injecting drug users. AIDS 1992; 6:1053-1068.

48. Estébanez P, Cifuentes I. Diferencias de riesgo por género para el VIII entre los UDVP (Parte I y II). Publicación oficial de la SEISIDA 1996: $1: 8-13$, y $2: 2-67$.
49. Doll DC. Tattoing in prison and HIV infection. Lancet 1988; 1:66-67.

50. Muller R, Stark K, Guggenmoos-Holzmann I, Wirth $\mathrm{D}$, Bienzle U. Imprisonment: a risk factor for HIV infection counteracting education and prevention programmes for intravenous drug users. AIDS 1995; 9(2):183-90.

51. Davies AG, Dominy NJ, Peters A, Bath GE, Burns SM, Richardson AM. HIV injecting drug uscrs in Edinburg: prevalence and correlates. J Acquir Defic Syndr Hum Retrovirol 1995; 8(4):399-405.

52. Eirós JM, Perlado E, Ortiz de Lejarazu R, Orduña A, Torres AR. Influencia de la antiguedad en la ADVP y la reclusión en la infección por VIH. Libro de Ponencias y Comunicaciones. I Congreso Nacional de Sanidad Penitenciaria. León; 1993. p. 340 .

53. Scano G, Luzi G, Mezzaroma I, Aiuti F, Vellucei A, Guastini L. et al. One year follow-up of HIV seropositive jail population. Allergol-Immunopathol (Madr) 1991; 19(4):165-6.

54. Medley G, Dolan KA, Stimson GV. A model of HIV transmission by siringe sharing in English prisons using surveys of injecting drug users. Eight International Conference on AIDS. Amsterdam, the Netherlands, 1992 (Oral abstract MoD0038).

55. Mutter RC, Grimes RM, Labarthe D. Evidence of intraprison spread of HIV infection. Arch Intern Med 1994; 154: 793-795.

56. Dolan K, Hall W, Wodak A, Gaughwin M. Evidence of HIV transmission in an Australian prison [letter]. Med J Aust 1994; 160:734.

57. Des Jarlais DC. The first and second decades of AIDS among injecting drug users. BJ Addictions 1992; 87:353-374.

58. Friedman SR. Des Jarlais DC. HIV among drug injectors: the epidemic and the response. AIDS Care 1991; 3:239-250.

59. Buning E, Van Brussel G. The efects of harm reduction in Amsterdam. Eur addict Res 1995; 1:92-98. 